ISSN-i 1390-7042 | ISSN-e 2602-8484

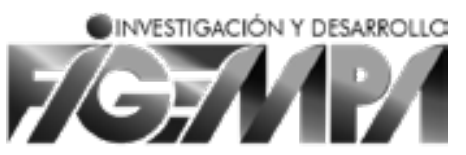

\title{
DISEÑO SOSTENIBLE DE UN PROCESO INDUSTRIAL LOCAL PARA LA OBTENCIÓN DE UNA BEBIDA HIDRATANTE DE HOJA DE TUNA
}

\section{Sustainable design of a local industrial process for obtaining a tuna leaf drink}

\author{
Mabel Parada Rivera \\ mparada@espoch.edu.ec \\ Escuela Superior Politécnica de Chimborazo \\ ORCID: https://orcid.org/0000-0001-7491-2078
}

\section{Sofía Godoy Ponce} sofia.godoy@espoch.edu.ec

Escuela Superior Politécnica de Chimborazo ORCID: https://orcid.org/0000-0002-6479-4343

\author{
Lourdes Carrera Beltrán \\ lcarreraboespoch.edu.ec \\ Escuela Superior Politécnica de Chimborazo \\ ORCID: https://orcid.org/0000-0002-0266-4893
}

Alexandra Tapia Borja alexandra.tapia@utc.edu.ec

Universidad Técnica de Cotopaxi

ORCID: https://orcid.org/0000-0001-6935-5211
Dayana Chávez Echeverría dayagabriela1@hotmail.com Investigadora Independiente ORCID: https://orcid.org/0000-0002-0482-6497
Recibido: septiembre de 2020

Aprobado: mayo de 2021 ARTÍ́CULO ORIGINAL

DOI: 10.29166/revfig.v11i1.3097

palabras Clave Diseño, proceso, sostenible, bebida, hidratante, formulación.

KEYWORDS Design, process, sustainable, drink, hydrating, formulation.

\section{RESUMEN}

En Ecuador se consume el fruto de la tuna, sin embargo, las hojas o cladodios son desechados sin aprovechar sus principales componentes. El presente estudio tiene como finalidad la elaboración de una bebida de hoja de tuna (Opuntia ficus-indica) por medio de un diseño de producción industrial sostenible en la localidad de Guano, para elaborar un producto de buena calidad, y que contribuya al desarrollo local del cantón. La caracterización socioeconómica se realizó a través de grupos focales y metodología social, la caracterización fisicoquímica de la materia prima y del producto final se realizó en un laboratorio particular. Los resultados muestran que la bebida de hoja de tuna tiene aceptación social, porque emplea como materia prima un componente local conocido por la población, cuyo proceso no genera impactos negativos. Su aceptación se basa en el hecho que, al ser procesada la hoja de tuna, ésta no pierde ninguno de sus componentes nutricionales, es un producto inocuo y con innumerables propiedades antioxidantes y desintoxicantes. El proyecto consta de un análisis socioeconómico y un proceso de industrialización de la bebida que consta de las siguientes etapas: recepción, inspección y selección de la materia prima, desespinado, lavado, mondado y despulpado, troceado, homogeneizado, agitado y mezclado, filtrado, pasteurizado, adición de aditivos, envasado y etiquetado. En el desarrollo experimental, se obtuvieron cuatro formulaciones para la bebida, al aplicar un análisis sensorial de jueces afectivos, se determinó que la formulación con sabor a durazno es la más aceptada en el grupo social de estudio.

\section{ABSTRACT}

In Ecuador, the fruit of the prickly pear is consumed, however, the leaves or cladodes are discarded without taking advantage of their main components. The purpose of this study is to prepare a prickly pear leaf drink (Opuntia ficus-indica) through a sustainable industrial production design for the town of Guano, producing a good quality product that contributes to local development of the canton. The socioeconomic characterization was carried out through focus groups and social methodology, while the physicochemical characterization of 
the raw material and the final product was carried out in a private laboratory. With the results, it was determined that this drink has social acceptance, because it uses as raw material a local component known to the population and whose process does not generate negative impacts. Its acceptance is based on the fact that, when the tuna leaf is processed, it does not lose any of its nutritional components, it is a harmless product with innumerable antioxidant and detoxifying properties. The project consists of a socioeconomic analysis and a process of industrialization of the drink that consists of the following stages: reception, inspection and selection of the raw material, deboning, washing, peeling and pulping, chopping, homogenizing, shaking and mixing, filtering, pasteurizing, adding additives, packaging and labeling. In the experimental development, four formulations for the drink were obtained, by applying a sensory analysis of affective judges, it was determined that the peach flavor formulation is the most accepted in the study social group.

\section{INTRODUCCIÓN}

A través de varios estudios de relación genética y morfológica de la especie opuntia ficus-indica, se rescata el hecho de que ésta es una de las de mayor importancia agronómica por sus rasgos evolutivos desde su origen y adaptación en los sistemas ecológicos (MAG, 2018). $\mathrm{Al}$ existir una correlación entre desempeño económico de productos con su diseño sostenible frente a retos ambientales y sociales, que demanda el modelo de desarrollo actual del país, Opuntia ficus-indica puede ser considerada un recurso de fácil acceso, producción $\mathrm{y}$ aprovechamiento, pues no requiere de condiciones estrictas para su desarrollo y adaptabilidad (Kiesling, 2010). De acuerdo a un estudio realizado por el Ministerio de Agricultura y Ganadería (MAG), el cultivo de tuna en el Ecuador ocupa aproximadamente 180 hectáreas de extensión (MAG, 2018; Kiesling, 2010). En el cantón Guano, alrededor de 600 agricultores se dedican al cultivo de tuna (Opuntia ficus-indica), que es, entre las cactáceas, la de mayor importancia agronómica, tanto por sus sabrosos frutos como por sus tallos que sirven de forraje o pueden ser consumidos como verdura (García Favela, 2013).

Para la disponibilidad de estos alimentos aptos para el consumo, se pondera especialmente el autoabastecimiento a través de la producción local (Diario El Día, 2016; Blanco-Navarro, 2010). Se destaca, implícitamente, el sistema de producción deseado para que los pequeños y medianos productores adopten las buenas prácticas agrícolas como el aprovechamiento de recursos, uso de los saberes ancestrales y el desarrollo de procesos, así como las tecnologías apropiadas para elevar la productividad local, a fin de evitar la dependencia de la producción del mercado internacional, reduciendo al mínimo los impactos ambientales negativos de los sistemas de producción y de consumo que constituye la finalidad de la pro- ducción sostenible (Ministerio del Ambiente, s. f.; Barazarte, 2017).

En el Ecuador se consume el fruto de la tuna, sin embargo, las hojas de esta fruta son desechadas sin que se aprovechen sus principales componentes, entre los que destacan: las proteínas, carbohidratos, hierro, calcio, grasas (Diario El Comercio, 2011). Este tipo de hojas son comparables por su valor nutricional a ciertas frutas y verduras como el melón, mango, uva, espinaca, alcachofa, berenjena, brócoli, respectivamente (Esquivel, 2004; ONU, 2001). Además, se señala que éstas poseen un porcentaje de agua de $94,14 \%$, bajo contenido de hidratos de carbono y proteínas, $2,5 \%$ y $1,98 \%$, respectivamente, contenido de fibra del $2 \%$, son ricas también en minerales como calcio $74,8 \mathrm{mg} / 100 \mathrm{~g}$ y hierro $0,86 \mathrm{mg} / 100 \mathrm{~g}$ y cuentan con un mínimo contenido de sodio, por lo que pueden ser consumidos por personas con problemas renales o de hipertensión (onU, 2018). También, contiene $12,6 \mathrm{mg} / 100 \mathrm{~g}$ de vitamina $\mathrm{C}$. El consumo de hoja de tuna aporta a la dieta solamente $27 \mathrm{kcal} / 100 \mathrm{~g}$, y debido a que contiene fibra soluble, su consumo ayuda a estabilizar la concentración de azúcar en la sangre (García, 2014).

La tuna también implica un beneficio económico, dado sus propiedades nutritivas y medicinales (Ochoa y Guerrero, 2013; Castro, 2009). La sostenibilidad de la producción de la tuna se centra en la economía rural y en las condiciones ambientales poco exigentes. Desde 2016, la mayoría de las empresas artesanales de la provincia de Chimborazo adquieren la tuna para procesarla artesanalmente y transformarla en productos para comercialización (Chávez, 2018). El fruto de la tuna es lo que mayormente se consume, dado que se desconocen los beneficios que proporcionan sus hojas, las cuales contienen gran cantidad de fibra, fotoquímicos, vitaminas, antioxidantes y minerales, im- 
pecables nutrientes para el cuerpo (Fagerholm et al., 2012; Sepúlveda y Rodríguez, 2003). Si se consideran los factores de adaptabilidad y las propiedades nutricionales que este vegetal posee se justifica la necesidad de industrializar la bebida de hoja de tuna para así ofrecerla al mercado como una alternativa saludable de consumo, opción que aprovecha en sí todos los componentes del vegetal (Sáenz, 2018), mediante un proceso artesanal con un diseño industrial para aumentar la producción de calidad, promoviendo la soberanía alimentaria de los ecuatorianos (Vicepresidencia de la República del Ecuador, 2015).

\section{MATERIALES Y MÉTODOS}

Para la consecución del presente proyecto se llevó a cabo el registro de parámetros de control y manipulación de variables objeto de estudio. Se utilizaron equipos e instrumentos, así como diversas técnicas de laboratorio para la caracterización de la materia prima y del producto terminado.

La metodología planteada para el presente estudio se dividió en 5 etapas:

- Etapa A: Análisis socioeconómico

- Etapa B: Caracterización de la materia prima

- Etapa C: Descripción del proceso de elaboración de la bebida de hoja de tuna

- Etapa D: Determinación del análisis sensorial de la bebida de hoja de tuna

- Etapa E: Validación del producto final

\section{ANÁLISIS SOCIOECONóMICO}

Para el análisis socioeconómico se realizó el estudio de costo-beneficio social del proceso y el posible impacto de éste.

El estudio socioeconómico comprendió aspectos relacionados con las condiciones socioculturales y socioeconómicas de la población de estudio, incorporando términos para condiciones de mejora de la población respecto a empleo, esperanza de vida, nivel de alfabetización, población económicamente activa. Se consideró el comportamiento de la comunidad respecto al grado de consumo de productos locales, el interés para proyectos sostenibles, permitiendo medir impactos en términos monetarios, sociales. Se realizó un estudio de las condiciones ambientales de la zona poblada para la definición de la capacidad de soporte del sector.

Se reconocieron cuatro etapas: identificación de la idea de bebidas sostenibles, prefactibilidad, factibilidad y, por último, la de diseño de una bebida bajo las condiciones detalladas.
Las variables socioeconómicas valoradas fueron la presencia de zonas agrícolas, afluentes y vertientes, zona poblada y formas de vida consideradas en torno al área de influencia directa e indirecta.

La muestra de trabajo para este análisis incluyó a los actores sociales de quienes surgió la idea inicial de producción de la bebida hidratante, la cual contó con el apoyo de quince socios vinculados con el GAD Municipal de Guano. Las pruebas socioeconómicas se desarrollaron con 62 miembros provenientes de catorce comunidades del cantón.

\section{CARACTERIZACIÓN DE LA MATERIA PRIMA}

Para la caracterización de los parámetros físico-químicos de la materia prima se siguió la metodología establecida por Sánchez y col. (2017) con algunas modificaciones. Parámetros tales como la biomasa fresca, longitud, ancho, grosor, acidez titulable (AT), $\mathrm{pH}$, contenidos de sólidos solubles totales (SST), ácido ascórbico, humedad, proteínas, cenizas, grasas, carbohidratos, pectina total, hierro, calcio y potasio se analizaron para $2 \mathrm{~kg}$ de muestra (Sánchez-Urdaneta, 2017).

\section{DESCRIPCIÓN DEL PROCESO DE ELABORACIÓN DE LA BEBIDA DE HOJA DE TUNA}

Mediante ensayos realizados a nivel de laboratorio, y al obtener cuatro distintas bebidas, de las cuales al ser evaluadas por jueces afectivos se escoge una de ellas: la bebida de hoja de tuna sabor a durazno, se establece que el mejor proceso industrial para la obtención de la bebida de hoja de tuna consiste en las siguientes etapas u operaciones: recepción de la materia prima en la cantidad necesaria, inspección y selección de la materia prima, desespinado, lavado, mondado y despulpado, troceado, homogeneizado, agitado y mezclado, filtrado, pasteurizado, adición de aditivos (conservante, edulcorante, saborizante), envasado y etiquetado, así como la formulación para la misma (ver Figura 1).

Para la elaboración de la bebida de hoja de tuna el proceso empieza con la recepción de la materia prima estimada con base en la caracterización fisicoquímica, gracias a la cual se determinará la calidad de la hoja de tuna para continuar con su procesamiento. Después se lleva a cabo la selección e inspección de la hoja de tuna (Espinoza, 2007), para separar el porcentaje de materia defectuosa, de la materia prima que esté en condiciones óptimas para el procesamiento. Se realiza la remoción de las espinas de la hoja de nopal, operación que facilita el posterior lavado de las hojas de tuna con una solución de hipoclorito de sodio al $5 \%(\mathrm{v} / \mathrm{v})$, para eliminar materiales extraños y la presencia 


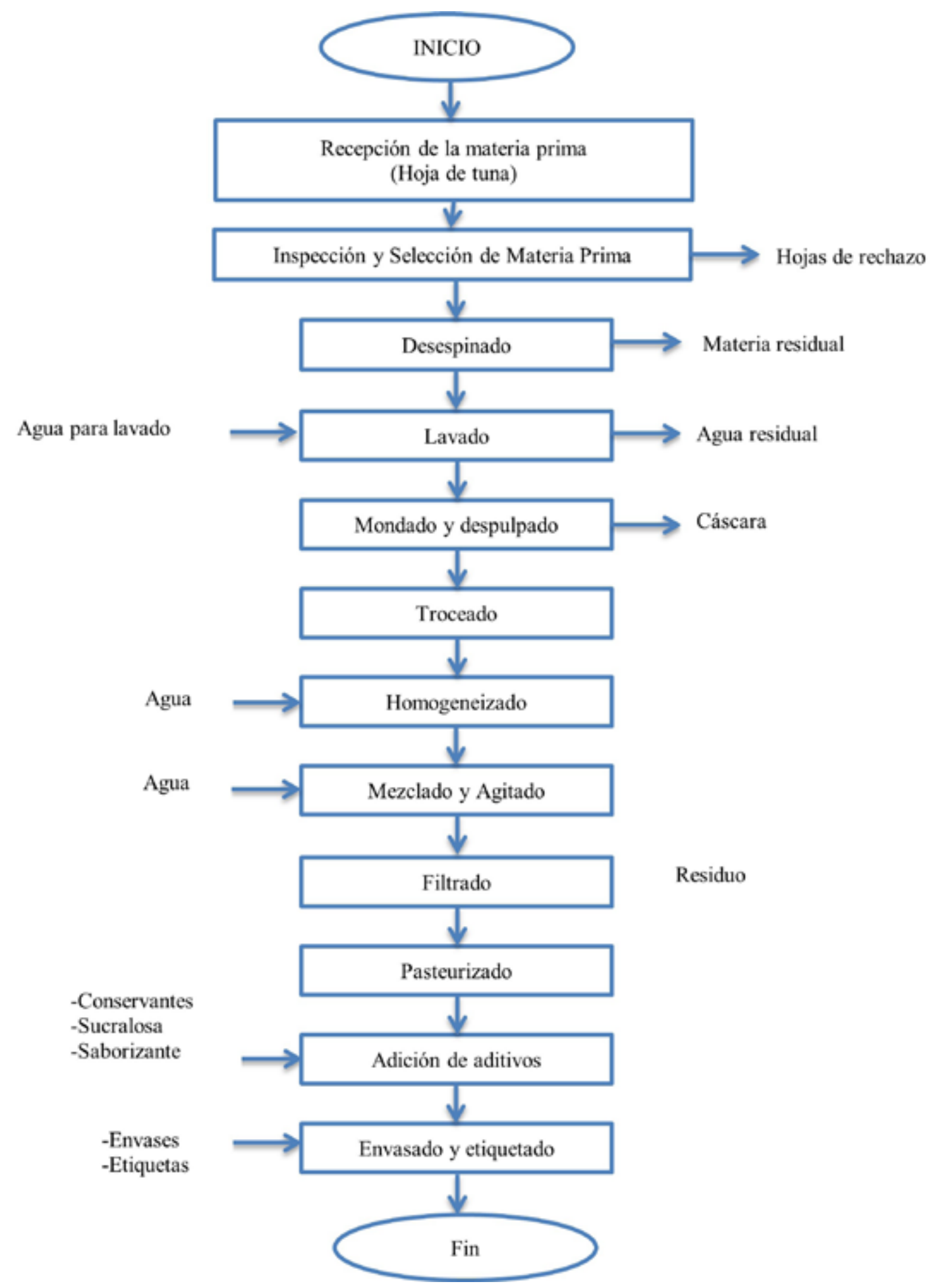

Figura 1. Diagrama de proceso para la elaboración de la bebida de hoja de tuna. Realizado por María Abarca (2018).

de microorganismos. A continuación, se realiza el mondado de la hoja de tuna, al retirar la corteza de la hoja se extrae la pulpa que ésta posee, cuando ya ha sido obtenida la pulpa de la hoja, se procede a trocearla con la ayuda de un cuchillo hasta obtener trozos pequeños de aproximadamente $3 \mathrm{~cm}$. Se licúa a $3600 \mathrm{rpm}$ en una licuadora industrial de acero inoxidable durante $1 \mathrm{~min}$ los cuadritos de la pulpa, para facilitar la homogeneización se añade solo un $34 \%$ del agua purificada total que debe llevar la mezcla. Se realiza el mezclado y agitado en un homogeneizador de la bebida de pulpa de hoja de tuna añadiendo un porcentaje de líquido de $66 \%$, es decir, el agua restante, durante un tiempo de 5 ho- 
ras. Se filtra y se obtiene una bebida con consistencia de gel. El tratamiento térmico se realiza en una marmita a una temperatura de $75{ }^{\circ} \mathrm{C}$, durante 30 segundos, inmediatamente después de transcurrido este tiempo se baja drásticamente la temperatura de la bebida hasta $\operatorname{los} 35^{\circ} \mathrm{C}$ con la finalidad de reducir la presencia de agentes patógenos y alargar la vida útil del producto. Después de obtenida la bebida, se adhieren a ésta los aditivos necesarios, esto se realiza después del tratamiento térmico para que dichos aditivos no se vean alterados por acción de la temperatura. Se utilizan las siguientes cantidades: $125 \mathrm{mg} /$ $\mathrm{kg}$ de benzoato de sodio, $125 \mathrm{mg} / \mathrm{kg}$ de sorbato de potasio, $0,5 \mathrm{ml} / \mathrm{L}$ de saborizante natural de durazno y $0,4 \mathrm{ml} / \mathrm{L}$ de sucralosa. Finalmente, la bebida de hoja de tuna se envasa en botellas de vidrio de 500 $\mathrm{ml}$, se las sella herméticamente y se las etiqueta.

\section{DETERMINACIÓN DEL ANÁLISIS SENSORIAL DE LA BEBIDA DE HOJA DE TUNA}

Se realizaron 4 ensayos diferentes con el objetivo de encontrar la bebida más adecuada para su procesamiento a nivel industrial, las cuales serán discriminadas según criterios sensoriales de sabor, color, aroma y consistencia (Rodríguez, 2017; Nazareno, 2013), como se detalla en la tabla 1 . Una vez culminado el proceso de evaluación del producto mediante las encuestas, se realiza la tabulación de datos, cálculo y análisis estadístico de los datos obtenidos (ver Tabla 1).

El análisis sensorial se realiza mediante el uso del método afectivo (Cordero, 2013), el cual consiste en la selección de un grupo de personas no entrenadas, denominadas «jueces afectivos», quienes forman parte de la población de estudio ya que se han considerado como consumidores directos o potenciales. La prueba se realizó el día 19 de octubre de 2018, en el Instituto Superior Tecnológico Carlos Cisneros, con la colaboración de 110 jueces afectivos. Una vez realizadas las encuestas se tabulan los resultados obtenidos en los que al observar los porcentajes la bebida con mayor aceptación es la bebida de hoja de tuna sabor a durazno. Además, se emplea la prueba chi-cuadrado para determinar la dependencia entre las variables, luego de obtenidos los resultados de la prueba chi-cuadrado para cada uno de los parámetros analizados y al realizar la respectiva comparación con el valor de chi-cuadrado crítico, se determina que para los parámetros sabor, color y aroma sí existe dependencia entre la muestra y el nivel de respuesta, mientras que para el parámetro consistencia no existe relación, lo cual concuerda debido a que la consistencia en las cuatro muestras analizadas es la misma (consistencia de gel).

\section{VALIDACIÓN DEL PRODUCTO FINAL}

Se realizó la validación del proceso mediante la caracterización físico-química del producto final de acuerdo con los parámetros que fueron analizados en la materia prima (hoja de tuna), y los análisis microbiológicos de acuerdo a la NTE INEN 2337:2008, jugos, pulpas, concentrados; néctares, bebidas de frutas y vegetales.

\section{RESULTADOS}

El grado de aceptación sobre el aprovechamiento de las hojas de la plantación de tuna surge por varias razones, entre éstas sobresale el hecho de que el cultivo de tuna es prominente en la zona que se caracteriza por tierras áridas, lo que convierte a la plantación en una especie local y cuyo costo-beneficio social es elevado, el bajo costo de producción, la demanda por productos innovadores y que promuevan la soberanía alimentaria establecida en la política pública nacional: Plan Nacional de Desarrollo Toda una Vida, y el impulso a la matriz productiva.

En la etapa de identificación de la idea se establecieron las razones bien fundadas para la aceptación o rechazo del proyecto. En la etapa inicial del proyecto se definió el alcance fundamentado en el nivel de aceptación o rechazo de la población hacia la propuesta, el resultado generado evidenció ausencia de rechazo, por lo que se adoptó la decisión de continuar con los análisis y la especificación de los estudios para la siguiente etapa.

La etapa de prefactibilidad generó como resultados las diferentes alternativas de sabor en la bebida de hoja de tuna obtenida, que fueron: piña, durazno y sabor natural, además se desarrolló el diseño y se ejecutó el análisis de todas ellas. La tercera etapa consistió en la precisión de la alternativa más viable, sus mecanismos de inversión, esquemas de desarrollo, protocolos de producción y la aceptación de la que se consideró como más apropiada para el objetivo.

Finalmente, la etapa de diseño generó la preparación de planos de ejecución bajo condiciones reales de la población de estudio, con sus posibles alternativas técnicas y económicas de solución.

La caracterización de la materia prima de la hoja de tuna, se detalla en la tabla 2 , en donde se observa que los parámetros analizados son los adecuados y correctos que requiere la materia prima para asegurar, en su procesamiento a nivel industrial, un producto de buena calidad y que cumpla con los parámetros establecidos según las normas respectivas (ver Tabla 2).

$\mathrm{Al}$ realizar la caracterización fisicoquímica se obtuvieron los siguientes resultados: $1,98 \%$ de proteína, $0,45 \%$ de grasa, $0,93 \%$ de cenizas, $96,44 \%$ de hume- 
Tabla 1. Codificación de las muestras de bebidas de hoja de tuna

\begin{tabular}{lll}
\hline Procedencia & Referencia & Código \\
\hline Bebida elaborada por Vita Tuna & Bebida elaborada artesanalmente & 1423 \\
Ensayos a nivel de laboratorio & Bebida natural sin saborizante & 1424 \\
& Bebida sabor a durazno & 1425 \\
& Bebida sabor a piña & 1426 \\
\hline
\end{tabular}

Tabla 2. Resultados de los análisis de caracterización fisicoquímica de la hoja de tuna

\begin{tabular}{llll}
\hline Parámetro & Unidades & Método de análisis & Valor determinado \\
\hline Proteína & $\%$ & INEN 1670 & 1,98 \\
Grasa & $\%$ & INEN 523 & 0,45 \\
Cenizas & $\%$ & INEN 401 & 0,93 \\
Humedad & $\%$ & INEN 1235 & 94,14 \\
Sólidos totales & $\%$ & INEN 1235 & 2,50 \\
Azúcares totales & ${ }^{\circ} \mathrm{Brix}$ & - & 2,50 \\
Ácido ascórbico & $\mathrm{mg} / 100 \mathrm{~g}$ & Volumétrico & 12,60 \\
Calcio & $\mathrm{mg} / 100 \mathrm{~g}$ & Volumétrico & 74,80 \\
Hierro & $\mathrm{mg} / 100 \mathrm{~g}$ & Colorimétrico & 0,86 \\
Acidez titulable & $\mathrm{mg} / 100 \mathrm{~g}$ & Volumétrico & 98,20 \\
pH & - & Potenciométrico & 5,60 \\
\hline
\end{tabular}

Fuente: Reportes por el laboratorio SAQMIC, 2019.

Tabla 3. Formulación porcentual de la bebida de hoja de tuna

\begin{tabular}{llll}
\hline Componente & Porcentaje & Cantidad & Unidad \\
\hline Pulpa de la hoja de tuna & $15,20 \%$ & 218,00 & $\mathrm{~kg}$ \\
Agua & $14,27 \%$ & 204,61 & $\mathrm{~L}$ \\
Benzoato de sodio & $0,17 \%$ & 2,38 & $\mathrm{~kg}$ \\
Sorbato de potasio & $0,17 \%$ & 2,38 & $\mathrm{~kg}$ \\
Sucralosa & $0,69 \%$ & 9,94 & $\mathrm{~L}$ \\
Tipos de saborizante (piña, durazno, natural) & $0,87 \%$ & 12,43 & $\mathrm{~L}$ \\
\hline
\end{tabular}

dad, 2,5\% de carbohidratos, $2,5^{\circ}$ Brix, $12,6 \mathrm{mg} / 100 \mathrm{~g}$ de vitamina C, 74,8 mg/100 g de Calcio, $0,86 \mathrm{mg} / 100$ $\mathrm{g}$ de hierro, 98,2 mg/100 g de acidez titulable y un pH de 5,6. No existe una norma que especifique los requisitos que los cladodios de nopal deban cumplir, sin embargo, al comparar estos resultados con los de análisis realizados en estudios anteriores con los valores obtenidos, estos poseen cifras aproximadas.
En la tabla 3 se detalla la cantidad de materia prima y aditivos necesarios para la formulación de la bebida de hoja de tuna a escala industrial, una vez realizados los distintos ensayos mediante el proceso de elaboración visto en el epígrafe anterior (ver Tabla 3.

En la tabla 4 se presentan los resultados del nivel de aceptación de la bebida por parte de los jueces afectivos una vez realizadas las encuestas. Se mues- 
tran resultados de frecuencia y los porcentajes obtenidos según cada muestra (ver Tabla 4).

Se establece que la muestra 1425 (bebida de hoja de tuna sabor a durazno) es la mejor bebida, debido a que presentó un mayor porcentaje de aceptación por los jueces afectivos.

Se valida el proceso mediante la caracterización fisicoquímica y microbiológica de la bebida de hoja de tuna (Guzmán y Chávez, 2007) que se detalla en las tablas 5 y 6 , respectivamente (ver Tablas 5 y 6 ).

$\mathrm{Al}$ realizar los análisis fisicoquímicos del producto final, se observa que la bebida aún presenta las propiedades nutritivas que posee la hoja de tuna inicialmente, además, los análisis microbiológicos denotan ausencia de coliformes totales, coliformes fecales, mohos y levaduras por lo que se comprueba la inocuidad del producto gracias a la asepsia con la que ha sido realizado su procesamiento.

Los resultados obtenidos mediante la caracterización fisicoquímica y microbiológica de la formulación según la norma técnica ecuatoriana NTE INEN 2337:2008, jugos, pulpas, concentrados; néctares, bebidas de frutas y vegetales, son proteína $0,10 \%$, ceniza $0,11 \%$, azúcares totales $0,41^{\circ} \mathrm{Brix}$, sólidos totales 0,74\%, hierro 0,60 mg/100 ml, calcio $12,8 \mathrm{mg} / 100 \mathrm{ml}$, grasa $0,12 \%$, acidez titulable $34 \mathrm{mg} / 100 \mathrm{ml}, \mathrm{pH} 5,3$, ácido ascórbico $34 \mathrm{mg} / 100 \mathrm{ml}$ y ausencia de coliformes fecales, coliformes totales, mohos y levaduras. Al comparar los valores obtenidos del producto final con los valores de los análisis de la caracterización fisicoquímica de la hoja de tuna (materia prima).

\section{DISCUSIóN}

La elaboración de una bebida hidratante con base en las hojas de tuna, bajo el concepto de sostenibilidad (variables ambientales como el aprovechamiento de recursos que no alteran la estructura y estabilidad del suelo, y que no generan la producción con alta concentración de fertilizantes, sociales y económicas) involucra un proceso impulsor del nuevo modelo de desarrollo del país, que se fundamenta en la endogeneidad del territorio, es decir, que promueve la producción local, considerando productos y materias primas propias del territorio, cuyo costo-beneficio social sea alto y cuyo impacto ambiental negativo sea el mínimo, es decir, cuyos efectos en la población local sean los esperados y no ocasionen condiciones adversas en todo lo que las etapas del proceso de extracción de materia prima y producción implica y, sobre todo, siempre bajo la rigurosidad de la normativa vigente (ARCSA, 2016).

La garantía de la eficiencia del diseño bajo las cuatro etapas empleadas para el estudio socioeconó- mico genera un carácter objetivo en el valor social, ambiental y económico, bajo una utilización óptima de los recursos. El estudio hace posible el aprendizaje constante; para el efecto se requiere experiencia, independencia e innovación. Este componente es la manera más viable en la priorización de las decisiones, para afianzar el beneficio de este tipo de proyectos, para financiarlos y para la búsqueda de estrategias de comunicación adecuadas con las partes interesadas.

En el proceso para la elaboración de una bebida de hoja de tuna, se parte de la selección de materia prima en la cual los resultados de sus análisis físico-químicos se obtuvieron los siguientes resultados: $1,98 \%$ de proteína, $0,45 \%$ de grasa, $0,93 \%$ de cenizas, $96,44 \%$ de humedad, 2,5\% de carbohidratos, 2,5 ${ }^{\circ}$ Brix, 12,6 mg/100 g de vitamina C (ácido ascórbico), $74,8 \mathrm{mg} / 100 \mathrm{~g}$ de calcio, $0,86 \mathrm{mg} / 100 \mathrm{~g}$ de hierro, 98,2 $\mathrm{mg} / 100 \mathrm{~g}$ de acidez titulable y un $\mathrm{pH}$ de 5,6. A pesar de que no existe una norma que especifique los requisitos que los cladodios de nopal deban cumplir, estos valores se aproximan a resultados de análisis bromatológicos realizados en un estudio previo sobre la composición química de las hojas de tuna en los cuales los valores se aproximan a los obtenidos en este estudio (Sánchez-Urdaneta, 2017).

Una vez realizados los distintos ensayos de la bebida se establece el proceso a nivel industrial, el diseño y los cálculos de ingeniería. Para lo cual se procesarán $218 \mathrm{~kg}$ de materia prima, que aproximadamente corresponden a 223 hojas de tuna, de los cuales se obtienen 275,67 L de bebida, después de la adición de agua y los distintos aditivos requeridos que fueron determinados anteriormente. Se continúa estableciendo los balances de masa y energía basados en los datos recolectados durante los ensayos realizados y una relación con los datos que se requieren para establecer el proceso a escala industrial.

Para la producción de la bebida de hoja de tuna se hicieron ensayos de tres diferentes muestras, en las cuales variaba el sabor. La primera muestra, bebida natural sin saborizante ni edulcorante; la segunda muestra, sabor a piña; y la tercera, sabor a durazno. Estas tres bebidas, junto con la bebida artesanal que se elabora en la empresa a pequeña escala, fueron evaluadas por 110 jueces afectivos mediante un análisis sensorial, obteniéndose una aceptación general de $9,09 \%, 24,55 \%, 60 \%$ y $6,36 \%$, respectivamente. De acuerdo con estos resultados, se estableció que la bebida a elaborar es la de sabor a durazno.

En los resultados del análisis físico-químico y microbiológico se obtuvo un porcentaje de proteína del $0,10 \%$, ceniza $0,11 \%$, azúcares totales $0,41^{\circ} \mathrm{Brix}$, sólidos totales $0,74 \%$, hierro $0,60 \mathrm{mg} / 100 \mathrm{ml}$, calcio 12,8 
Tabla 4. Resultados estadísticos del nivel de aceptación de la bebida por los jueces afectivos

\begin{tabular}{lllll}
\hline Código & Frecuencia & Porcentaje (\%) & Porcentaje válido (\%) & Porcentaje acumulado (\%) \\
\hline 1425 & 66 & 60,00 & 60,00 & 60,00 \\
1426 & 27 & 24,55 & 24,55 & 84,55 \\
1424 & 10 & 9,09 & 9,09 & 93,64 \\
1423 & 7 & 6,36 & 6,36 & 100 \\
Total & 110 & 100 & 100 & \\
\hline
\end{tabular}

Tabla 5. Resultados de los análisis fisicoquímicos de la bebida de hoja de tuna

\begin{tabular}{llll}
\hline Determinaciones & Unidades & Método & Resultado \\
\hline Proteína & $\%$ & INEN 16 & 0,10 \\
Ceniza & $\%$ & INEN 401 & 0,11 \\
Azúcares totales & ${ }^{\circ}$ Brix & - & 0,41 \\
Sólidos totales & $\%$ & INEN 1235 & 0,74 \\
Hierro & $\mathrm{mg} / 100 \mathrm{ml}$ & colorimétrico & 0,60 \\
Calcio & $\mathrm{mg} / 100 \mathrm{ml}$ & volumétrico & 12,80 \\
Grasa & $\%$ & INEN 12 & 0,12 \\
Acidez titulable & $\mathrm{mg} / 100 \mathrm{ml}$ & volumétrico & 34,00 \\
pH & - & potenciométrico & 5,30 \\
Ácido ascórbico & $\mathrm{mg} / 100 \mathrm{ml}$ & volumétrico & 34,00 \\
\hline
\end{tabular}

Fuente: Reportes del laboratorio SAQMIC, 2019.

Tabla 6. Resultados del análisis microbiológico de la bebida de hoja de tuna

\begin{tabular}{llllll}
\hline Parámetro & Unidad & Min. & Máx. & Método & Resultado \\
\hline Coliformes totales & $\mathrm{UFC} / \mathrm{ml}$ & $<3$ & - & NTE INEN 1529-6 & Ausencia \\
Coliformes fecales & $\mathrm{UFC} / \mathrm{ml}$ & $<3$ & - & NTE INEN 1529-8 & Ausencia \\
Mohos y levaduras & $\mathrm{UFC} / \mathrm{ml}$ & $<10$ & 10 & NTE INEN 1529-10 & Ausencia \\
\hline
\end{tabular}

Fuente: Reportes del laboratorio SAQMIC, 2019.

$\mathrm{mg} / 100 \mathrm{ml}$, grasa $0,12 \%$, acidez titulable $34 \mathrm{mg} / 100$ $\mathrm{ml}, \mathrm{pH} 5,3$, ácido ascórbico $34 \mathrm{mg} / 100 \mathrm{ml}$ y ausencia de coliformes fecales, coliformes totales, mohos y levaduras, por lo tanto se establece que la bebida posee propiedades nutritivas por su contenido de proteínas, minerales como hierro, calcio y vitamina C. Además, se evidencia la inocuidad del producto.

\section{CONCLUSIONES}

El análisis socioeconómico permitió establecer la base diagnóstica del diseño, para implantar estrategias innovadoras que permitan rentabilidad social, ambiental y económica, de manera que no se afecten la población local y los beneficiarios directos del proceso de producción. 
La caracterización físico-química de la materia prima, hoja de tuna (Opuntia ficus-indica), muestra resultados similares a los obtenidos en otros estudios, posibilitando su utilización como bebida de consumo.

La bebida de hoja de tuna, sabor a durazno, se establece como el mejor proceso industrial para la obtención de una bebida de consumo general, debido a que posee características organolépticas que agradan al potencial consumidor.

Los valores de la caracterización fisicoquímica y microbiológica de la hoja de tuna y el producto final indican las propiedades nutritivas y la inocuidad de la bebida, pudiendo determinarse que está apta para el consumo humano.

\section{REFERENCIAS}

ARCSA. 2016. Normativa técnica sanitaria para alimentos procesados. Disponible en: http://www.competencias. gob.ec/wp-content/uploads/2017/06/o6NOR2016-REsoluciono3-1.pdf. (Consultado: 18 de agosto de 2018).

Barazarte, H. 2017. Características físicas y químicas de cladodios de Opuntia ficus-indica (L.) Mill. Disponible en: https://www.researchgate.net/publication/315746700_Caracteristicas_fisicas_y_quimicas_de_cladodios_de_Opuntia_ficus-indica_L_Mill. (Consultado: 10 de septiembre de 2018).

Blanco-Navarro, M. 2010. Nopal (Opuntia ficus indica; L.), delicioso y medicinal. La Calera, 10(14), 71-72. https:// doi.org/10.5377/calera.v10i14.316.

Castro, J. 2009. Cultivo de tuna. Disponible en: http://www. agrolalibertad.gob.pe/sites/default/files/MANUAL\%20 TECNICO\%20DE\%2OTUNA.pdf (consultado: 28 de agosto de 2018).

Chávez, D. 2018. Diseño del proceso industrial para la obtención de una bebida de hoja de tuna (Opuntia ficus-indica) para la empresa Vita Tuna. Escuela Superior Politécnica de Chimborazo.

Cordero, G. 2013. Aplicación del análisis sensorial de los alimentos en la cocina y en la industria alimentaria. Sevilla: Universidad Pablo de Olavide.

Diario El Comercio. 2011. La tuna, cuatro variedades se producen en el país. Disponible en: https://www.elcomercio. com/actualidad/negocios/tuna-cuatro-variedades-se-producen.html. (Consultado: 21 de septiembre de 2018).

Diario El Día. 2016. La tuna y sus innumerables propiedades. Disponible en: https://eldia.com.do/la-tuna-y-sus-innumerables-propiedades/. (Consultado: 20 de agosto de 2018).

Espinoza, J. 2007. Evaluación sensorial de los alimentos. La Habana-Cuba Universitaria.

Esquivel, P. 2004. Los frutos de las cactáceas y su potencial como materia prima. Agronomía Mesoamericana, 15(2), 215-219. Disponible en : https://www.redalyc.org/articulo. oa?id=43715212. (Consultado: 15 de Agosto de 2018).

Fagerholm, N., Käyhkö, N., Ndumbaro, F., Khamis, M. 2012. Community stakeholders' knowledge in landscape assessments-Mapping indicators for landscape services.
Ecol Indic. 1(18), 421-33.

García Favela, B. 2013. Mucílago de nopal (Opuntia spp.) sobre propiedades micromorfológicas y estructurales del suelo en trigo (Tesis). Universidad de Chapingo. México.

García, M. 2014. Análisis sensorial de alimentos. Disponible en: https://www.uaeh.edu.mx/scige/boletin/icbi/n3/ m1.html (Consultado: 21 de septiembre de 2018).

Guzmán, D. y Chávez, J. 2007. Estudio bromatológico del cladodio del nopal (Opuntia ficus-indica) para el consumo humano. Scielo 73(1). Disponible en: http:// www.scielo.org.pe/scielo.php?script=sci_arttext\&pi$\mathrm{d}=$ S1810-634X2007000100005. (Consultado: $12 \mathrm{de}$ agosto de 2018)

Kiesling, R. 2010. Origen, domesticación y distribución de Opuntia ficus-indica. Disponible en: https://www. researchgate.net/publication/228607157_Origen_domesticacion_y_distribucion_de_Opuntia_ficus-indica. (consultado: 14 de agosto de 2018).

Ministerio de Agricultura y Ganadería. 2018. La producción de tuna, una actividad rentable. Disponible en: https:// www.agricultura.gob.ec/la-produccion-de-tuna-una-actividad-rentable/ (consultado: 14 de agosto de 2018).

Ministerio del Ambiente. (s. f.) Guía de buenas prácticas ambientales.

Nazareno, M. 2013. Actas de la Segunda Reunión para el Aprovechamiento Integral de la Tuna y otras Cactáceas y I Reunión Sudamericana CACTUSNET FAO-ICARDA. FAO.

Ochoa, C. y Guerrero, J. 2013. Efecto de la temperatura de almacenamiento sobre las características de la calidad de tuna blanca Villanueva (Opuntia albicarpa). Revista Iberoamericana Tecnología Postcosecha, 14(2), 149-61. Disponible en: http://www.redalyc.org/articulo. oa?id=81329290008 (Consultado: 14 de septiembre de 2018)

Organización de las Naciones Unidas para la Agricultura y la Alimentación. 2001. Perfiles nutricionales por países-Ecuador. Roma: FAO.

Organización de las Naciones Unidas para la Alimentación y la Agricultura. 2018. Cultivos tradicionales, nopal. Disponible en: https://www.fao.org/traditional-crops/ cactuspear/es/. (Consultado: 24 de agosto de 2018).

Rodríguez, C. 2017. Evaluación del mucílago de nopal (Opuntia ficus-indica) como agente estabilizante en néctar de maracuyá (Passiflora edulis). Disponible en: https:// ciencia.lasalle.edu.co/ing_alimentos. (Consultado: 05 de agosto de 2018).

Sáenz, C. 2018. Utilización agroindustrial del nopal. Disponible en: http://repositorio.uchile.cl/bitstream/handle/2250/120301/Utilizacion agroindustrial-del-nopal. pdf? sequence $=1$. (Consultado 14 de agosto de 2018).

Sánchez-Urdaneta, A. 2017. Características físicas y químicas de cladodios de Opuntia ficus-indica (L.) Mill. Revista Fac. Agronomía, 34(2), 175-86. Disponible en: https:// produccioncientificaluz.org/index.php/agronomia/article/ view/27227. (Consultado: 20 de septiembre de 2018).

Sepúlveda, S. y Rodríguez, A. 2003. El enfoque territorial del desarrollo rural. Disponible en: https://books.google. com.ec/books/about/El_enfoque_territorial_del_desa- 
rrollo_ru.html?id=1tOB3FlFHUgC\&printsec=frontcover\&source=kp_read_button\&redir_esc $=\mathrm{y} \# \mathrm{v}=$ onepage\&q\&f=false. (Consultado: 14 de septiembre de 2018).

Vicepresidencia República del Ecuador. 2015. Estrategia nacional para el cambio de la matriz productiva. Disponi- ble en: https://www.vicepresidencia.gob.ec/wp-content/ uploads/2013/10/ENCMPweb.pdf. (Consultado: 20 de septiembre de 2018). 